\title{
Isolated Intramedullary Spinal Rosai-Dorfman Disease in a Child: A Case Report
}

\author{
Amgad Mohamed Abdelhady Moussa ${ }^{1}$ Mohamed Ismail Degheidy ${ }^{1}$ Noha Mohamed Osman ${ }^{1}$ \\ Sara Makkeyah ${ }^{2}$ Shaimaa Abdel Sattar ${ }^{1}$
}

${ }^{1}$ Department of Radiodiagnosis, Faculty of Medicine, Ain Shams University, Cairo, Egypt

${ }^{2}$ Department of Pediatric, Hematology-Oncology Unit, Faculty of Medicine, Ain Shams University, Cairo, Egypt

\begin{abstract}
Address for correspondence Amgad Mohamed Abdelhady Moussa, MBBCh, MSc, Department of Radiology, Ain Shams University, 5 Obour Buildings, Salah Salem St., Heliopolis, Cairo, Egypt 11371 (e-mail: amgadmoussa89@gmail.com).
\end{abstract}

J Child Sci 2019;9:e7-e10.

\author{
Abstract \\ Keywords \\ - Rosai-Dorfman \\ disease \\ - histiocytic disorder \\ - intramedullary spinal \\ cord masses
}

Rosai-Dorfman disease (RDD) is an uncommon benign histiocytic proliferative disorder commonly involving the cervical lymph nodes and, less frequently, extranodal sites. The histological hallmark of RDD is emperipolesis displayed by lesional histiocytes. Central nervous system involvement is rare and usually intracranial; intramedullary spinal involvement is even less common and, to our knowledge, rarely reported in children. Herein, we report a case of RDD with isolated intramedullary spinal involvement in a child, which, to our knowledge, is the first case reported in an infant.

\section{Introduction}

Rosai-Dorfman disease (RDD), also known as sinus histiocytosis with massive lymphadenopathy, is an uncommon benign histiocytic proliferative disorder which was named after J. Rosai and R. F. Dorfman in 1969. ${ }^{1,2}$ However, an extensive search of the literature done by Gaitonde revealed that it was first reported by Destombes in $1965 .^{3,4}$ Although RDD has been reported in all age groups, it most frequently presents in children and young adults, with a slight male predominance, ${ }^{5}$ and it is mainly observed in individuals of African and European descent rather than in Asian populations. ${ }^{6}$ It is generally characterized by bilateral, painless, massive cervical lymphadenopathy. In around $30 \%$ of cases, extranodal involvement can be found, including the skin, orbit, upper respiratory tract, and bones. ${ }^{7}$ Central nervous system (CNS) involvement has been described but is uncommon and is often intracranial. It usually manifests as duralbased masses, and intraparenchymal involvement is less common. Intramedullary spinal cord involvement is rare. ${ }^{8}$ We present an extremely rare case of isolated intramedullary spinal RDD. To our knowledge, there are only a handful of cases described in children, and this is the first case described in an infant.

received

November 2, 2018 accepted after revision January 28, 2019

\section{Case Report}

An 18-month-old previously healthy girl presented with forward head tilt, progressive cervical kyphosis, and difficulty in walking with frequent falling. She had no history of preceding trauma or infection and no constitutional symptoms. Family history was significant for breast cancer in her paternal grandmother and CNS tumors in the paternal side. Physical examination revealed a well appearing child with no skin rashes, abnormal pigmentation, or palpable lymphadenopathy. Her cardiac, respiratory, and abdominal examinations were normal. Neurologically, she had left lower limb weakness Grade 2/5, with Brisk reflexes, normal upper limbs and right lower limb examination, and no cranial nerve affection. Her laboratory investigations showed white blood cells $8.6 \times 10^{3} / \mu \mathrm{L}$, hemoglobin 13.3 $\mathrm{g} / \mathrm{dL}$, platelets $277 \times 10^{3} / \mu \mathrm{L}$, erythrocyte sedimentation rate $10 \mathrm{~mm} / \mathrm{hr}$, immunoglobulin A $85 \mathrm{mg} / \mathrm{dL}$, immunoglobulin M $167 \mathrm{mg} / \mathrm{dL}$, and immunoglobulin G $731 \mathrm{mg} / \mathrm{dL}$.

Cervical magnetic resonance imaging (MRI) was done before referral to our institution revealing an oval, welldefined, intramedullary soft tissue lesion exerting fusiform cord expansion extending from C6 to D3 level surrounded by proximal and distal cord edema. The lesion displays

Copyright $\odot 2019$ Georg Thieme Verlag License terms KG Stuttgart · New York
DOI https://doi.org/ 10.1055/s-0039-1683452. ISSN 2474-5871. 


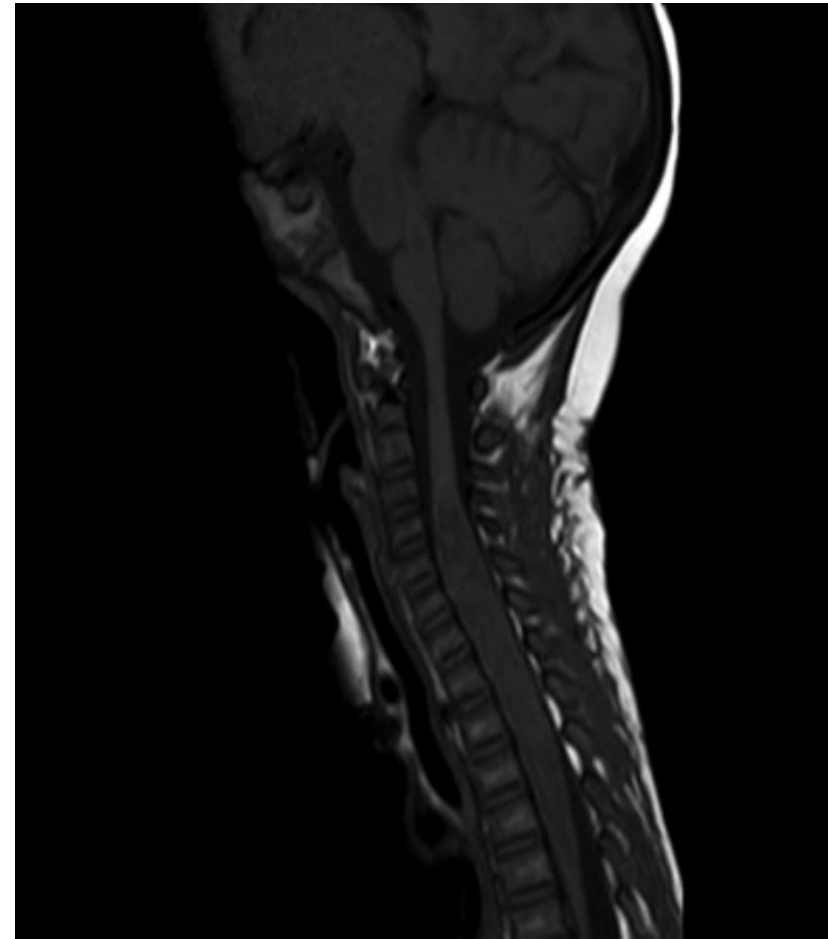

Fig. 1 Sagittal T1-weighted image showing intermediate intensity spinal cord fusiform swelling extending from C6 to D3 level.

relatively intermediate signal on $\mathrm{T} 1$, as well as on $\mathrm{T} 2$ and short tau inversion recovery-weighted images and shows homogenous avid post-contrast enhancement on $\mathrm{T} 1$ postcontrast images (-Figs. 1-3). The lesion displayed no calcifications, necrosis, or cystic changes. The top differential

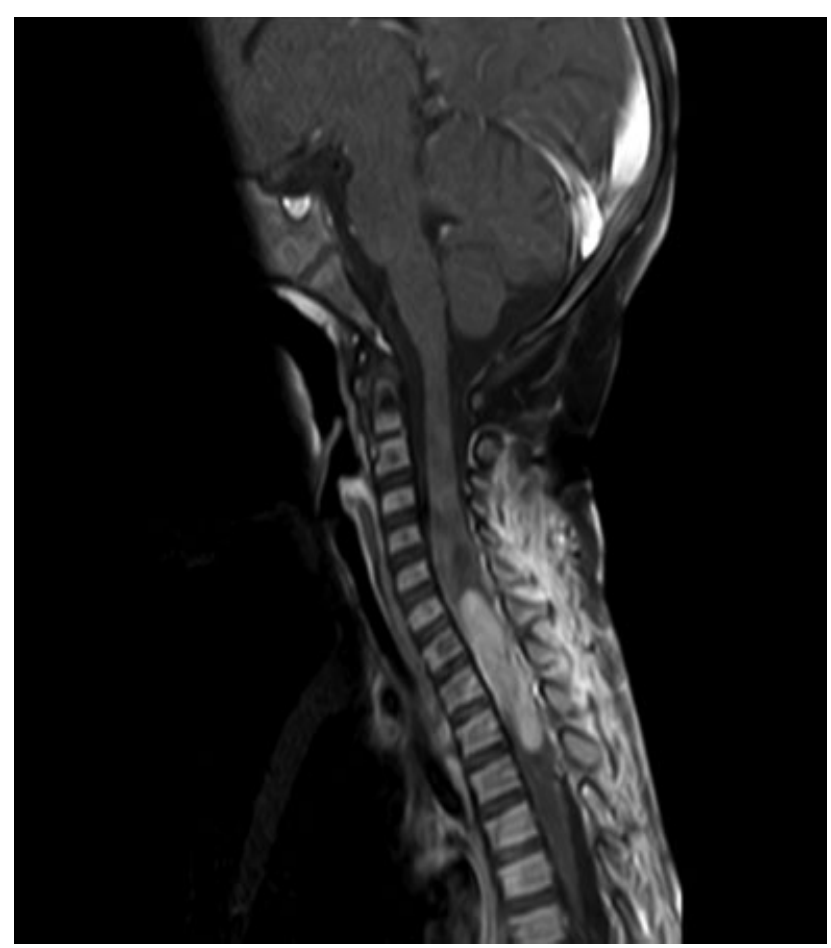

Fig. 2 Sagittal T1-weighted post-contrast image showing avid homogenous enhancement of a well-defined intramedullary oval lesion with better delineation of its extent.

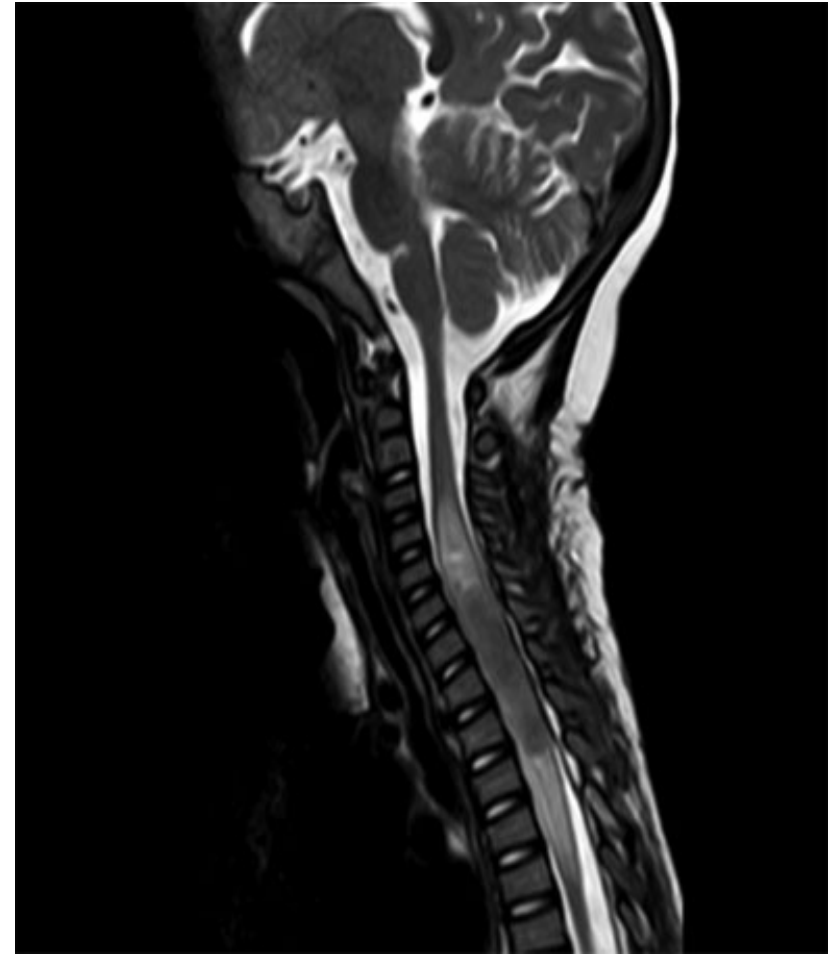

Fig. 3 Sagittal T2-weighted image showing the isointense intramedullary spinal cord lesion with increased signal intensity in the proximal and distal cord denoting spinal cord edema.

diagnoses postulated were intramedullary astrocytoma, ependymoma, and ganglioglioma. Surgical intervention was done for trial of mass excision, but only open biopsy was performed. Histopathological examination revealed polymorphic population formed of lymphocytes, excess eosinophils, together with histiocytic aggregates having vesicular nuclei and eosinophilic cytoplasm. However, there was no evidence of emperipolesis. Moderate vascularity was also seen. Immunohistochemical staining was positive for protein $\mathrm{S} 100$ and CD68 and negative for CD1a, GFAP, and CD34. This picture was suggestive of RDD. However, further immunohistochemical and molecular analysis to confirm the diagnosis was not done due to logistical issues.

The patient was started on steroid treatment to decrease spinal cord edema with minimal improvement. One month later, a repeat cervical MRI was done showing similar findings with decreased spinal cord edema.

\section{Discussion}

Classical sporadic RDD typically involves lymph nodes. ${ }^{2,9}$ Extranodal disease is apparent in approximately $40 \%$ of all cases, and most commonly involves the skin, retro-orbital tissue, nasal cavity, bone, and soft tissue. ${ }^{10}$ RDD of the nervous system has been reported in less than $5 \%$ of all cases, most of which were in the brain. Involvement of the spine has been described in only $20 \%$ of those with CNS involvement, with the vast majority of cases being related to the Dura. ${ }^{11}$ A retrospective analysis showed that 210 cases of CNS involvement were reported ever since the description of 
the disease, of which only 24 were isolated spinal RDD. Most cases of isolated spinal RDD were extramedullary duralbased lesions; only two cases were intramedullary. ${ }^{8}$ Moreover, in children, isolated intramedullary spinal RDD is extremely rare and has only been reported twice in two 12 years old children, making our case the first reported in an infant. $^{7,12}$

The diagnosis of RDD cannot depend on imaging as there are no pathognomonic features. ${ }^{1}$ CNS RDD usually presents with dural-based lesions; and that along with their homogenous enhancement on post-contrast T1-weighted images leads to their frequent misdiagnosis as meningiomas. On T1weighted images, RDD usually appears as a single or multiple well-defined lesions with isointense or hyperintense signal that shows distinct and homogenous contrast enhancement. ${ }^{13}$ On T2-weighted images, RDD usually displays isointense signal, yet lesions with hypo- and hyperintense signal have been reported. ${ }^{1,13}$ Heterogeneous hypointense signal on T2-weighted images has been reported for numerous cases and has been proposed to be used as a differentiating point between RDD and meningiomas, which are commonly iso- or hyperintense on T2-weighted images. Calcifications are rarely seen in RDD. ${ }^{5}$

RDD is a disease of nonmalignant histiocytes that infiltrate lymph nodes or extranodal tissues. Definitive diagnosis can only be made by histological analysis of the affected tissues. The association between emperipolesis, which is the nondestructive phagocytosis of lymphocytes or erythrocytes, and a typical immunohistochemical pattern characterized by positivity for S-100 protein and CD68 antigen and negativity for CD1a antigen is diagnostic for RDD. ${ }^{14}$ In cases where emperipolesis is not described, preventing the confirmatory diagnosis of RDD, Langerhans cell histiocytosis (LCH) is included in the differential diagnosis as it is also positive for S-100 protein. However, $\mathrm{LCH}$ is often also positive for the CD1a antigen. ${ }^{15}$ Further immunohistochemical and molecular analysis can be used to add strength to the diagnosis, such as the detection of $B R A F$ mutations, which are seen in almost two-thirds of $\mathrm{LCH}$ cases and rarely seen in RDD. ${ }^{16}$ Our case showed the typical immunohistochemical characteristics; however, emperipolesis was not an evident feature and further immunohistochemical and molecular analysis was not done due to logistical issues.

Laboratory abnormalities are nonspecific with elevated erythrocyte sedimentation rate and leukocytosis, high ferritin, hypergammaglobulinemia, and autoimmune hemolytic anemia. $^{17}$

The etiology of RDD is unknown and it is considered an idiopathic histiocytosis. Links to human herpes virus, parvovirus B19, and Epstein-Barr virus have been made, but none have been confirmed so far. ${ }^{18}$ Clonal nature of some RDD patients has been proven, specifically those that show KRAS and MAP2K1 mutations, but this is only seen in about onethird of patients. ${ }^{19}$

Sporadic RDD is often self-limited with a good outcome, especially in classical form. However, 5 to $11 \%$ of patients may die due to their disease. ${ }^{17}$ Because of the critical impact on spinal elements, and the magnitude of the symptoms, surgery is usually the optimal approach in these manifestations of RDD. ${ }^{20}$ Steroid treatment has shown some therapeutic benefit in a few reported cases with CNS involvement. ${ }^{8}$ In our case, it led to a decrease in spinal cord edema on follow-up imaging done 1 month later with no significant clinical benefit to the patient.

\section{Conclusion}

Isolated spinal intramedullary RDD is an extremely rare disease, and the diagnosis is overwhelmingly difficult. Imaging characteristics still vary between cases and immunohistochemistry is still the only reliable basis for diagnosis. Further imaging studies need to be continued for better characterization of RDD spinal and brain lesions, thus guiding adequate curative management.

Conflict of Interest

None declared.

\section{References}

1 Sciacca S, Barkas K, Heptinstall L, McNamara C, Shetty R. RosaiDorfman disease with spinal cord compression: a diagnostic challenge. Eur Spine J 2015;24(Suppl 4):S529-S535

2 Rosai J, Dorfman RF. Sinus histiocytosis with massive lymphadenopathy. A newly recognized benign clinicopathological entity. Arch Pathol 1969;87(01):63-70

3 Gaitonde S. Multifocal, extranodal sinus histiocytosis with massive lymphadenopathy: an overview. Arch Pathol Lab Med 2007; 131(07):1117-1121

4 Destombes P. [Adenitis with lipid excess, in children or young adults, seen in the Antilles and in Mali. (4 cases)]. Bull Soc Pathol Exot 1965;58(06):1169-1175

5 Kozak B, Talbott J, Uzelac A, Rehani B. Rosai-Dorfman disease isolated to the thoracic epidural spine. J Radiol Case Rep 2015;9 (11):6-16

6 Liu G, Wang H, Yang Z, Tang T, Zhang S. Is it a metastatic disease: a case report and new understanding of Rosai-Dorfman disease? Am J Dermatopathol 2016;38(06):e72-e76

7 Huang BY, Liu HL, Yu CJ. Isolated intramedullary spinal RosaiDorfman disease: a case report and literature review. World Neurosurg 2016;88:694.e11-694.e15

8 Sandoval-Sus JD, Sandoval-Leon AC, Chapman JR, et al. RosaiDorfman disease of the central nervous system: report of 6 cases and review of the literature. Medicine (Baltimore) 2014;93(03): 165-175

9 Destombes P, Destombes M, Martin L. [Pseudotumoral lymph node lipidic histiocytosis. Further case in a young Martinique woman]. Bull Soc Pathol Exot 1972;65(03):481-488

10 Vaiselbuh SR, Bryceson YT, Allen CE, Whitlock JA, Abla O. Updates on histiocytic disorders. Pediatr Blood Cancer 2014;61(07): 1329-1335

11 Rocha-Maguey J, Felix-Torrontegui JA, Cabrera-López M, Gutiérrez-Castro M, Montante-Montes de Oca D. A new case of cervical intramedullary sinus histiocytosis causing paraplegia and review of the literature. Surg Neurol Int 2016;7:9

12 Yao K, Li TF, Zhu MW, et al. An intramedullary cervical cord lesion in a 12-year-old girl. Neuropathology 2013;33(05):582-585

13 Huang BY, Zhang H, Zong WJ, Sun YH. Rosai-Dorfman disease of rare isolated spinal involvement: report of 4 cases and literature review. World Neurosurg 2016;85:367.e11-367.e16 
14 di Dio F, Mariotti I, Coccolini E, Bruzzi P, Predieri B, Iughetti L. Unusual presentation of Rosai-Dorfman disease in a 14-monthold Italian child: a case report and review of the literature. BMC Pediatr 2016;16:62

15 Vadivelu S, Mangano FT, Miller CR, Leonard JR. Multifocal Langerhans cell histiocytosis of the pediatric spine: a case report and literature review. Childs Nerv Syst 2007;23(01):127-131

16 Badalian-Very G, Vergilio JA, Degar BA, et al. Recurrent BRAF mutations in Langerhans cell histiocytosis. Blood 2010;116(11): 1919-1923

17 Emile JF, Abla O, Fraitag S, et al; Histiocyte Society. Revised classification of histiocytoses and neoplasms of the macro- phage-dendritic cell lineages. Blood 2016;127(22): 2672-2681

18 Dalia S, Sagatys E, Sokol L, Kubal T. Rosai-Dorfman disease: tumor biology, clinical features, pathology, and treatment. Cancer Contr 2014;21(04):322-327

19 Garces S, Medeiros LJ, Patel KP, et al. Mutually exclusive recurrent KRAS and MAP2K1 mutations in Rosai-Dorfman disease. Mod Pathol 2017;30(10):1367-1377

20 El Molla M, Mahasneh T, Holmes SE, Al-Khawaja D. Rare presentation of Rosai-Dorfman disease mimicking a cervical intramedullary spinal cord tumor. World Neurosurg 2014;81(02):442. e7-442.e9 